\title{
Tierisch gut! Pflanzen- und Tierbeziehungen im Palmengarten
}

\author{
Hilke STEINECKE
}

Von Januar bis März 2010 gab es eine neue Nachtführungs-Reihe im Palmengarten mit dem Titel „Tierisch gut!“. An 15 Abenden führten wir unsere Gruppen durch das nächtliche Tropicarium, begegneten dabei echten Tieren und Modellen sowie Pflanzen, die Symbiosen oder andere Beziehungen zu bestimmten Tieren eingehen.

Begonnen wurde im Eingangsoktogon an der Weltkugel. Unsere Gäste erfuhren, welche Unterschiede es zwischen Tieren und Pflanzen gibt und dass die autotrophen Pflanzen über die Nahrungskette Lebensgrundlage sowohl für Pflanzenals auch für Fleischfresser sind. Dementsprechend viele Strategien haben Pflanzen entwickelt, Fraßfeinde vom Verzehr ihrer Blätter abzuhalten, sei es durch Tarnung oder durch stechende Dornen und Stacheln. Andererseits sind viele Pflanzen zur Bestäubung oder Ausbreitung ihrer Samen auf Tiere angewiesen, weshalb viele raffinierte Anlockungstricks entstanden sind.

Tiere kommen auf verschiedenen Wegen in das Tropicarium: Einige werden bewusst angesiedelt, weil sie eine Besucherattraktion sind oder Schädlinge vertilgen. Andere werden ohne Einwilligung des Palmengartens von Besuchern ausgesetzt, oder sie nisten sich selbst in den Gewächshäusern ein. So brütet beispielsweise regelmäßig eine Amsel unter Glas, oder ein Eichhörnchen zieht seine Jungen im Savannenhaus auf. Die vielen kleinen Pflanzenschädlinge wie Blattläuse oder Spinnmilben sind bei den Gärtnerinnen und Gärtnern nicht gerade beliebt, weshalb sie ganz spezielle Nützlinge wie z. B. den Australischen Marienkäfer züchten und in den befallenen Beständen aussetzen.

Die Form mancher Pflanzen wird mit Tieren in Verbindung gebracht, was sich in vielen Pflanzennamen offenbart wie z. B. Elefantenfuß, Kängurupfote, Löwenohr, Froschlöffel, Fledermausblume und Schlangenknöterich. Für viele war es faszinierend, eine kleine, 19 Mio. Jahre alte versteinerte Meergrundel in den Händen zu halten.
Dieser fossile Fisch stammt aus einer Baugrube neben dem Palmengarten und lebte einst in einem Ausläufer der Ur-Mittelmeeres, der auch das Gelände des heutigen Palmengartens bedeckte.

Nach einer kleinen Stärkung mit musikalischer Untermalung durch das mexikanische Lied "La Cucaracha" (die Küchenschabe) ging es zunächst in das Halb- und Nebelwüsten-Haus. Tiere und Pflanzen in Trockengebiete müssen mit extrem wenig Wasser auskommen können. Beispiele hierfür waren Oryx-Antilope und Wüstenschildkröte, die uns in der Nebelwüste in Form eines Schädels bzw. Skelettes begegneten. Lebende Steine (Lithops) verstecken sich vor durstigen oder hungrigen Tieren, indem sie sich in Form und Färbung dem steinigen Untergrund anpassen und somit nahezu unsichtbar werden. Ähnliche Strategien zeigen in Amerika auch diverse kleinere Kakteen, von denen Beispiele vorgeführt wurden. Spannend ist auch die Dreiecksbeziehung in der afrikanischen Savanne zwischen Akazien, Giraffen und Ameisen. Die Akazien entwickeln spezielle hohle Dornen, in denen die Ameisen leben können. Die Ameisen erhalten von der Akazie Nahrung, die von Blattnektarien abgegeben wird. Als Gegenleistung patrouillieren die Ameisen auf der Akazie und schützen sie so vor Schädlingen. Giraffen streifen mit ihrer langen, klebrigen Zunge die Akazienblätter ab, die Dornen halten sie nur wenig vom Fressen ab. Wenn Giraffen die Akazien nicht befressen, bilden die Bäume weniger Dornen, aber auch weniger Nektarien. Dadurch wird der Anreiz für die Ameisen geringer, auf der Akazie zu patroullieren. Als Resultat werden die Bäume bei der Abwesenheit der Giraffen stärker von Schädlingen angegriffen und wachsen schlechter.

Die afrikanische Savanne ist durch viele Großtiere charakterisiert, die größten sind die Elefanten. Sie nehmen täglich etwa $150 \mathrm{~kg}$ pflanzliche Nahrung auf. Ein großer Teil davon wird wieder 
ausgeschieden, weshalb Elefantendung entsprechend strohig-faserig ist, ähnlich wie Pferdeäpfel. In Afrika und Indien wird der kaum stinkende Elefantendung zur Herstellung von $\mathrm{Pa}$ pier genutzt. Unsere Gäste konnten anhand von Original-Elefantendung aus dem Zoo und Elefantenpapier-Kladden den Grad der Veredelung dieses Naturproduktes nachvollziehen. Zum Vergleich mit dem frischen Dung wurde garantiert nicht stinkender Kot zum Bestaunen ausgegeben, nämlich mehrere Millionen Jahre alte Koprolithen (versteinerter Kot) von Krokodil und Schildkröte.

Gleich nebenan wurde im Dornwald-Haus an einer Uncarina (Pedaliaceae) Station gemacht. Ihre mit Widerhaken ausgestatteten Früchte verfangen sich im Fell oder an den Füßen von Tieren und werden somit ausgebreitet. Weitere Beispiele für Pflanzen mit extrem stechend-hakigen Früchten aus der Verwandtschaft von Uncarina sind Teufelskralle (Harpagophytum procumbens) und Gamshornfrucht-Pflanze (Proboscidea lousianica). Auf der weiteren Route gab es Eukalyptus zu sehen und Spannendes zum Nahrungsspezialisten Koalabär (Phascolarctos cinereus) zu erfahren. Als Anpassung an die schwer verdauliche Nahrung aus Eukalyptusblättern ist sein Blinddarm etwa 2,5 m lang. Der Koala kann das Wasser in den Eukalyptusblättern extrem gut ausnutzen, weshalb er so gut wie kein Wasser trinken muss. Das haben bereits die Aborigines beobachtet. Der Name Koala stammt aus einer Aborigines-Sprache und bedeutet so viel wie „ohne Wasser“ bzw. „ohne zu trinken“. Neben dem zukünftigen Australien-Beet vor dem Wasserfall durfte ein Känguru natürlich nicht fehlen. Je nachdem, ob es sich um Wald- oder Steppenkängurus handelt, sind die Pflanzenfresser auf unterschiedliche Nahrungspflanzen spezialisiert. Zum Streicheln war an dieser Station ein echtes Kängurufell deponiert.

Abb. 1 (oben): Die purpurroten Kaktusfrüchte werden von Vögeln gefressen, die später die Samen an einem anderen Ort wieder ausscheiden.

Abb. 2 (unten): Die winzigen, im Tropicarium lebenden Pfeiffrösche stimmen im Dunkeln ihre Pfeifrufe an.
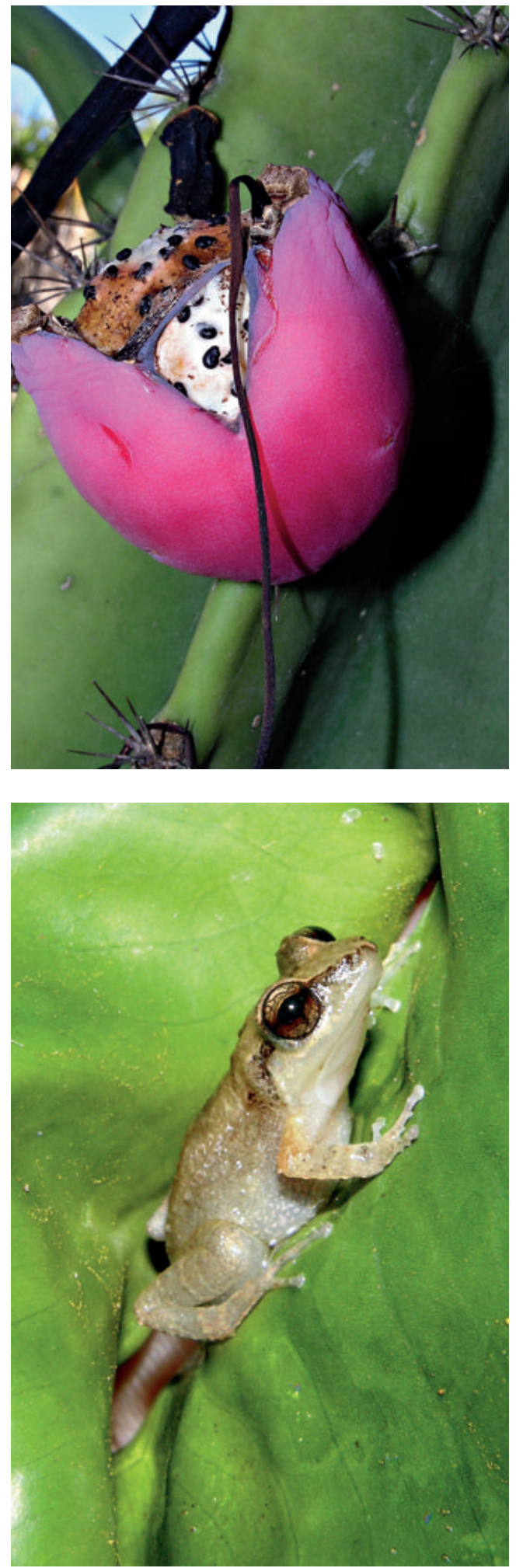


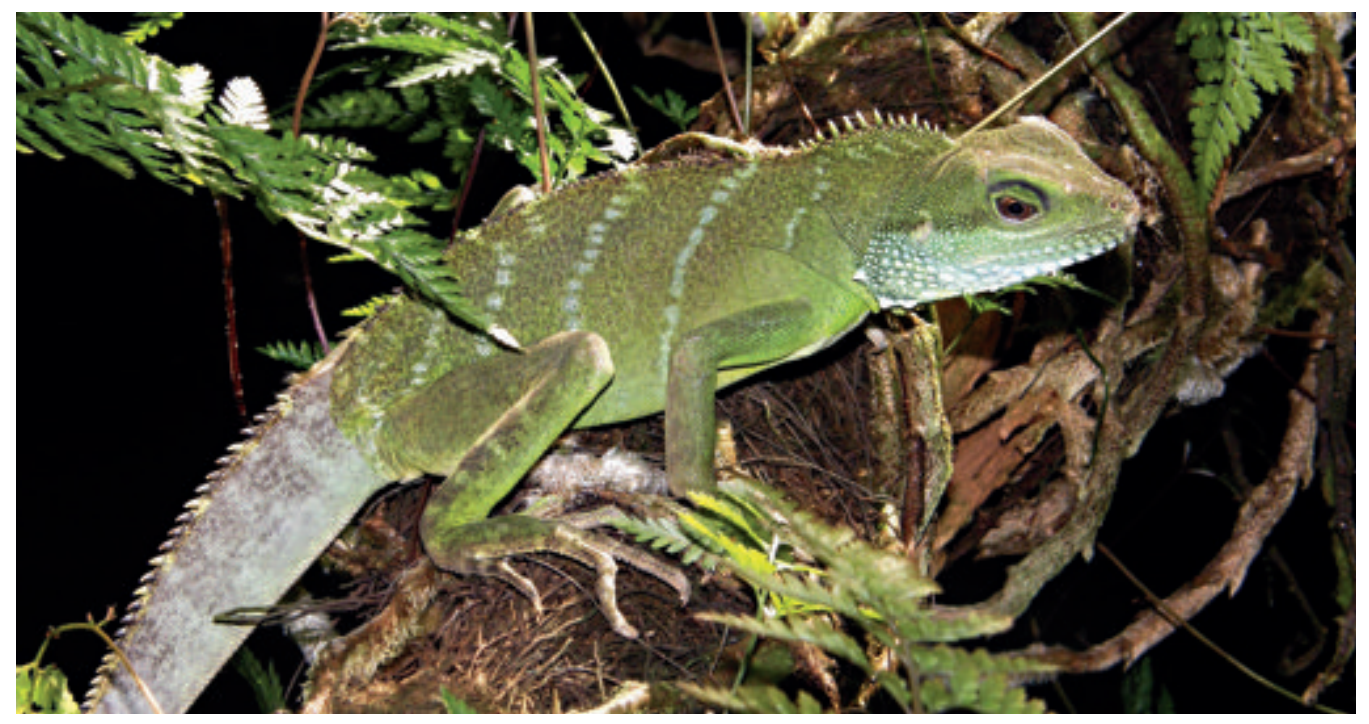

Im Nordteil des Tropicariums, der den feuchten Tropen gewidmet ist, können verschiedene Tiere beobachtet werden, darunter besonders zahlreich die großen Schaben, die vor allem im Dunkeln unterwegs sind. Mit etwas Glück hüpfen im Dunkeln gelegentlich auch Kröten und Gewächshausheimchen über den Weg oder sitzt eine smaragdgrüne Wasseragame wie ein kleiner Drache auf Lauer. Die Grüne Wasseragame (Physignathus cocincinus) stammt aus Südostasien. Sie kann bis zu $100 \mathrm{~cm}$ groß werden, allerdings entfallen davon zwei Drittel auf die Schwanzlänge. Weibchen bleiben etwas kleiner. Wasseragamen ruhen gern auf Ästen über Wasserflächen, von denen sie sich bei Gefahr ins Wasser fallen lassen. Im Tropicarium ernähren sich die Wasseragamen vermutlich vorwiegend von Insekten wie Schaben oder Würmern, es kann gelegentlich aber auch schon mal eine Maus unter den Beutetieren sein.

Allgegenwärtig sind auch die vielen kleinen tropischen Ameisen, die in vielen Blüten auf Nektarsuche gehen. In den Tropen gibt es viele Pflanzen, die mit oft ganz speziellen AmeisenArten eine Symbiose eingehen. Im Monsun-Passat-Haus wurde zu diesem Thema u. a. der Ameisenbaum (Cecropia) vorgestellt. Seine gekammerten Stämme sind hohl. Deren Wände sind über den Blattansätzen sehr dünn und werden von Ameisen der Gattung Azteca durchbis- sen. Die Kammern werden dann als Wohnraum verwendet. Cecropia bildet an der Blattstielbasis protein- und fettreiche Futterkörperchen (MÜLLERsche Körperchen), die besonders von den Azteca-Ameisen-Larven genutzt werden. Als weitere Ameisenpflanzen wurden Myrmecodia (Rubiaceae) und Schomburgkia (Orchidaceae) mit ihren für Ameisen hohlen Knollen bzw. Pseudobulben vorgestellt. Eine auf Ameisen (Camponotus schmitzi) hoch spezialisierte Art ist auch die Kannenpflanze Nepenthes bicalcarata. Die Ameisen leben in zerstreuten Kolonien in speziellen, ausgehöhlten Verdickungen im Übergangsbereich von Ranke zu Kanne. Die Ameise ernährt sich hauptsächlich von Nektar, den die Kanne über zwei dornenartige Nektarien ausscheidet. Es war beeindruckend, sich von den stechenden Eigenschaften dieser Nektarien zu überzeugen. Während normalerweise Insekten, die einmal in die Kanne geraten sind, ihr nicht mehr entfliehen können und verdaut werden, taucht Camponotus schmitzi sogar in die Flüssigkeit der Kanne ab und ernährt sich von der Beute der Kanne.

Weiter ging es zu unserer „Kaffee-Plantage“ im Nebelwald-Haus. Die roten Kaffeekirschen wer-

Abb. 3: Wasseragame, gut getarnt zwischen Pflanzen an ihrem Stammplatz im Tropicarium. 
den in Indonesien von einer Schleichkatze, dem Fleckenmusang (Paradoxurus hermaphroditus) gefressen. Die von den Tieren wieder ausgeschiedenen Kerne („Kaffeebohnen“) werden gesammelt und zu einem ganz besonderen Kaffeegetränk (Kopi Luwak) aufgearbeitet. Solch ein Kaffee hat natürlich seinen Preis, in Europa wird $1 \mathrm{~kg}$ dieser angedauten Kaffeebohnen mit etwa 1000 Euro gehandelt. Aus Ermangelung dieses teuren Kaffees wurden eine andere kleine KaffeeSpezialität angeboten.

Sehr teuer sind auch Koi (Brokatkarpfen), eine Zuchtform des Karpfens (Cyprinus carpio), wenn sie ganz bestimmte Zeichnungen tragen. Verschiedene orange, schwarz und weiß gemusterte Exemplare konnten im Wasserbecken des Nebelwaldes beobachtet werden. Da die wertvollsten Koi aus Japan kommen, gehen viele davon aus, dass dort auch ihre Heimat sei. KoiKarpfen stammen jedoch aus China und aus dem Kaspischen Meer und wurden früher auch als Speisefische gehalten. Mit Längen um $70 \mathrm{~cm}$ werden sie deutlich größer als Goldfische. Durch ihre zwei Barteln am Maul können Koi leicht von Goldfischen unterschieden werden. Bei einem Abstecher in das Bromelien-Haus konnten wassergefüllte Blattzisternen erkundet werden. Im natürlichen Lebensraum werden diese kleinen Wasserbasins von diversen Tieren, Insektenlarven, Kaulquappen, Frösche etc. als Lebensraum genutzt. Während der jahreszeitlich späteren Führungen, als es draußen schon wieder etwas wärmer war, begannen in diesem Haus auch die kleinen karibischen Pfeiffrösche der Gattung Eleutherodactylus mit ihrem nächtlichen Balzkonzert. Die unscheinbaren, graubraunen, nur etwa $2 \mathrm{~cm}$ großen Tiere sind zwar weit $\mathrm{zu}$ hören, aber optisch so gut getarnt, dass sie nur schwer zu finden sind. Pfeiffrösche sind unproblematisch in Gewächshäusern zu halten, selbst wenn es kein stehendes Wasser gibt: während ihrer Entwicklung reifen direkt kleine Frösche heran, die aus den Eiern schlüpfen, das wasserabhängige Kaulquappenstadium wird übersprungen.

Die letzte Station war das Mangrove-Haus. Unter der Kokospalme überraschte eine (nicht lebende) Gruppe aus verschiedenen Affen. Dazu erklang das Lied von der geklauten Kokosnuss. Im Lied hat das Affenbaby die Kokosnuss geklaut. In Polynesien sagt man aber einem Krebs (Birgus latro) nach, dass er Kokosnüsse stehle. Der als Palmendieb bezeichnete Land-Einsiedlerkrebs klettert gelegentlich tatsächlich auf Palmen und schält die grünen Kokosfrüchte, um an den nahrhaften Kern zu gelangen, den er mit gezielten Schlägen seiner Scheren öffnen kann. Wenn sich bei dieser Aktion eine Kokosnuss löst und vom Baum fällt, passiert das zufällig und wird nicht bewusst vom Krebs verursacht. Da es passieren kann, dass man unglücklicherweise von einer herabfallenden Kokosnuss erschlagen wird, die von einem Palmendieb gelöst wurde, wird der Krebs gelegentlich auch "widow maker" (Witwenmacher) genannt.

Im Tropicarium lauert glücklicherweise keine Gefahr wie in der Natur. Im kleinen Reisfeld gibt es auch keine extrem giftigen Aga-Kröten (Bufo marinus). Mit einer Körperlänge bis $22 \mathrm{~cm}$ und einem Gewicht von mindestens $1 \mathrm{~kg}$ gehören sie zu den größten Froschlurchen der Welt. Sie stammen aus Amerika und wurden in verschiedenen Gebieten der Erde in Zuckerrohrplantagen zur biologischen Schädlingsbekämpfung eingesetzt, so auch in Australien. In Australien stellt die Aga-Kröte heute ein extrem großes ökologisches Problem dar. Da sie hier keine natürlichen Feinde hat, vermehrt sie sich extrem schnell und verdrängt dabei die konkurrenzschwächeren heimischen Amphibien aus ihrem Lebensraum. Es gibt Kampagnen, die Aga-Kröten-Bestände zu verringern, was aber sehr schwer und kaum durchführbar ist. Seit einiger Zeit wird das Leder der Aga-Kröte zu verschiedenen Produkten verarbeitet, was zumindest einen Anreiz darstellt, die Tiere vermehrt zu fangen. Auf der Führung wurde als Demonstrationsobjekt eine Aga-Kröten-Ledergeldbörse gezeigt, in der man „Kröten“ aufbewahren kann. Mit großem Interesse ging dieses weiche Lederobjekt durch die Hände der Teilnehmenden.

Während des langen Winters war es anschließend ein großer Kontrast, nach zwei Stunden die angenehme, tropische Wärme in den Gewächshäusern gegen die eisige Kälte draußen im Garten einzutauschen. 